\title{
Representaciones sociales del autoexamen de mama en mujeres adultas de zonas rurales de Risaralda
}

Santiago Builes Robayo'; Edwar Albeiro Galeano Rodas'; Jhon Jairo Henao Arias'; Juan Pablo Marín González’; Pablo Emilio Mora Jaramillo’; Lenny Sandri Perea Mosquera'; Katheryn Salazar Londoño'; Julián David Vargas Marín'

Docentes Asesores: José Alonso Andrade Salazar², Tatiana Rodríguez Amador ${ }^{3}$

RESUMEN Esta investigación tiene como objetivo explorar las Representaciones Sociales de un grupo de mujeres de la zona rural de Risaralda, acerca del autoexamen de mama como práctica de autocuidado frente a la detección temprana del cáncer, $\mathrm{y}$ entender la dinámica del interaccionismo comunitario en cuanto a los determinantes de las prácticas de esta población. Para el anterior estudio se realizará una investigación de tipo cualitativo exploratorio, durante el segundo semestre de 2019, con mujeres que no han sido diagnosticadas con algún tipo de cáncer.

PALABRAS

Cáncer de Mama;

CLAVE Autocuidado;

Prevención;

Representaciones Sociales. 


\title{
Social representations of breast self-examination in adult women in rural areas of Risaralda
}

\begin{abstract}
This research aims to explore the Social Representations of a group of women from rural Risaralda, about breast self-examination as a self-care practice in the face of early cancer detection, and understand the dynamics of community interactionism in terms of the determinants of the practices of this population. For the previous study, an exploratory qualitative research will be carried out during the second half of 2019 with women who have not been diagnosed with some type of cancer.
\end{abstract}

Breast Cancer;

KEYWORDS Self-care;

Prevention;

Social Representations. 


\section{INTRODUCCIÓN}

Según la Organización Mundial de la Salud (OMS), el cáncer es un proceso de crecimiento y diseminación incontrolada de células, que puede aparecer prácticamente en cualquier lugar del cuerpo (1). A nivel de la mama, este se define como la proliferación anormal y desordenada de células mamarias malignas que conducen al crecimiento descontrolado de un tumor dentro de ella, el cual tiene la capacidad de invadir a otros órganos (2).

En América, cada año más de 462,000 mujeres son diagnosticadas con cáncer de mama, y casi 100,000 mueren a causa de esta enfermedad (3). Para Colombia, el fenómeno del cáncer es un problema de salud pública progresivo, siendo el cáncer de mama uno de los más comunes en la población femenina adulta, según los datos de la cuenta de Alto Costo, correspondientes a la población afiliada al Sistema General de Seguridad Social en Salud (SGSSS), en el periodo comprendido entre el 02 de enero de 2015 al 01 de enero de 2016, el cáncer de mama ocupó el primer lugar entre los tumores malignos más frecuentes en la población general, y entre las mujeres un total de 43.846 pacientes afectadas (4).

En el país, para el periodo comprendido entre el 2 de enero de 2016 y el 1 de enero de 2017, la prevalencia del cáncer de mama fue de 198,3 por 100.000 habitantes mujeres, Los departamentos con la mayor prevalencia, superior a la nacional, fueron Risaralda, Antioquia, Quindío, Bogotá, D.C., Valle del Cauca. En cuanto al departamento de Risaralda, El SGSS, reportó en el año 2017, una prevalencia de 309,5 y una tasa de mortalidad ajustada de 14.1 por 100.000 habitantes mujeres (5).

Lo anterior, materializa las estimaciones de la OMS, que apuntan a que la incidencia de cáncer de mama está aumentando, lo cual puede deberse a distintas razones, dentro de las cuales se destacan una mayor esperanza de vida, el aumento de la urbanización y la adopción de modos de vida occidentales (6).

Las causas o factores de riesgo del cáncer de mama pueden evidenciarse en la figura 1.

Esta información deja entrever que los factores de riesgo son totalmente actuales y pertinentes, donde quizás la mayoría de las mujeres contemporáneas se pueden identificar con uno o más de ellos.

En cuanto a las estrategias de prevención, aunque estas reducen en cierta medida el riesgo, no pueden eliminar la mayoría de los casos de cáncer de mama que se dan en los países de ingresos bajos y medios, donde el diagnóstico del problema se hace en fases muy avanzadas. Es así como la detección precoz se encuentra encaminada a mejorar el pronóstico y la supervivencia de esos casos y sobre todo en esos países, con el propósito de conocer los primeros signos y síntomas, basado en la exploración clínica de las mamas (6). 


\begin{tabular}{|c|c|c|c|}
\hline Factores reproductivos & Factores hormonales & $\begin{array}{l}\text { Factores nutricionales y } \\
\text { de modo de vida }\end{array}$ & Otros factores \\
\hline $\begin{array}{l}\text { Primera menstruación a edad } \\
\text { temprana + }\end{array}$ & $\begin{array}{l}\text { Uso de } \mathrm{AO} \text { (actual frente a } \\
\text { ninguno) }\end{array}$ & $\begin{array}{l}\text { Obesidad ( }>30 \text { IMC frente a } \\
<25 \text { ) } \\
\text { Premenopáusica - } \\
\text { Posmenopáusica + }\end{array}$ & $\begin{array}{l}\text { Antecedentes familiares (madre } \\
\text { o hermana) }{ }^{4+++}\end{array}$ \\
\hline $\begin{array}{l}\text { Edad al nacer el primer hijo } \\
(>35 \text { años frente } a<20)++\end{array}$ & $\begin{array}{l}\text { Restitución de estrógenos } \\
\text { (más de } 10 \text { años frente a } \\
\text { ninguno) }\end{array}$ & $\begin{array}{l}\text { Aumento de peso en la edad } \\
\text { adulta } \\
\text { (posmenopáusico) ++ }\end{array}$ & $\begin{array}{l}\text { Antecedentes familiares (familiar } \\
\text { en primer grado) }{ }^{b}++\end{array}$ \\
\hline $\begin{array}{l}\text { Número de nacimientos } \\
\text { ( } 0 \text { frente a } 1 \text { hijo }+\end{array}$ & $\begin{array}{l}\text { Restitución de estrógenos y } \\
\text { progesterona } \\
\text { (más de } 5 \text { años frente a } \\
\text { ninguno) ++ }\end{array}$ & $\begin{array}{l}\text { Consumo de alcohol ( } 1 \text { o más } \\
\text { bebidas al dia frente a ninguna) } \\
+\end{array}$ & $\begin{array}{l}\text { Ascendencia judia } \\
\text { (si frente a no) + }\end{array}$ \\
\hline $\begin{array}{l}\text { Edad de aparición de la } \\
\text { menopausia } \\
\text { (incremento de } 5 \text { años) }\end{array}$ & $\begin{array}{l}\text { Concentración elevada de } \\
\text { estrógenos o andrógenos en } \\
\text { sangre (postmenopausia) +++ }\end{array}$ & $\begin{array}{l}\text { Estatura (>5 pies con } 7 \\
\text { pulgadas) }+\end{array}$ & $\begin{array}{l}\text { Radiación ionizante } \\
\text { (si frente a no) + }\end{array}$ \\
\hline $\begin{array}{l}\text { Lactancia materna } \\
\text { ( }>1 \text { año frente a ninguno) - }\end{array}$ & $\begin{array}{l}\text { Concentración elevada de } \\
\text { prolactina en sangre ++ }\end{array}$ & $\begin{array}{l}\text { Actividad fisica (>3 horas por } \\
\text { semana) - } \\
\text { Grasas monosaturadas }{ }^{t}- \\
\text { (frente a grasas saturadas) } \\
\text { Bajo consumo de frutas y } \\
\text { verduras }{ }^{c} \text { - } \\
\text { (especialmente en el cáncer de } \\
\text { mama positivo para receptores } \\
\text { de estrógenos) + }\end{array}$ & $\begin{array}{l}\text { Enfermedad mamaria benigna } \\
\text { (diagnosticada por un médico) } \\
++ \\
\text { Densidad mamaria } \\
\text { (categoría superior frente a la } \\
\text { inferior) +++ }\end{array}$ \\
\hline
\end{tabular}

Nota: IMC, indice de masa corporal; $A O$, anticonceptivos orales; + , riesgo relativo $(R R)=1,1$ a 1,$4 ;++, R R=1,5$ a 2,$9 ;+++, R R=3,0$ a 6,$9 ;-, R R=$ 0,7 a 0,8 .

'Dos familiares en primer grado con antecedentes de cáncer de mama antes de los 65 años por comparación con ningún familiar.

* Familiar en primer grado con antecedentes de cáncer de mama antes de los 65 años por comparación con ningún familiar. ' Cuartil superior (primer 25\%) frente al cuartil inferior (el 25\% más bajo).

${ }^{0}$ Enfermedad quistica o fibroquistica crónica reconocida clinicamente u otra enfermedad mamaria benigna frente a ninguna.

\section{Figura 1.}

Factores de riesgo del cáncer de mama (7)

Los efectos que surgen tras el diagnóstico del cáncer de mama no solo significan una amenaza de muerte para muchas mujeres que lo padecen, sino que implica, para la mayoría, una sentencia de soledad existencial plagada de sufrimientos orgánicos y de desmoronamiento progresivo de su integridad física, asociados a un aislamiento afectivo y espiritual, forzado por la incomprensión, el prejuicio y las propias angustias que sufren quienes tienen relación con ellas (8).

Lo expuesto, permite visualizar un panorama donde el diagnóstico temprano del cáncer de mama, se ratifica como una de las estrategias más importantes para disminuir el número de muertes por este factor, y de manera particular para los países y poblaciones de ingresos bajos y medios, donde la enfermedad se diagnostica en fases avanzadas y los recursos son muy limitados, más aún cuando se evidencia que las prácticas de autocuidado, como el autoexamen, el examen clínico y la mamografía, no se realizan frecuentemente, así las mujeres conozcan sobre el tema 
y las consecuencias de este(9). También se trata de corroborar lo expresado por la OMS, donde establece que los mensajes de concientización sobre el cáncer de mama son más eficaces si son apropiados desde el punto de vista sociocultural (7).

En cuanto a los referentes teóricos, se encuentra que las representaciones sociales (RS) son un sistema teórico elaborado por Serge Moscovici, el cual se relaciona con la construcción social de la realidad; el autor las define como "un conjunto de conceptos, declaraciones y explicaciones originadas en la vida cotidiana. En el curso de las comunicaciones interindividuales. Equivalen, en nuestra sociedad, a los mitos y sistemas de creencias de las sociedades tradicionales, puede incluso, afirmarse que son la versión contemporánea del sentido común" (10).

Para Serge Moscovici (1979), estas representaciones sociales conservan subjetividad, constituidas por un "repertorio de conceptos, explicaciones, creencias y actitudes que se van originando en la vida diaria, por lo tanto, son compartidas socialmente (...) pueden entenderse como la aplicación de categorías que las personas utilizan para interpretar o comprender situaciones, y que se caracterizan por ser rígidas y no poner en tela de juicio sus propias aseveraciones" (p. 73) (11).

Las RS forman entonces sistemas cognitivos, en los cuales es posible reconocer la presencia de estereotipos, opiniones, creencias, valores y normas, las cuales pueden tener una orientación actitudinal positiva o negativa. Se constituyen a su vez como sistemas de códigos, valores, lógicas clasificatorias, principios interpretativos y orientadores de las prácticas, que definen la llamada conciencia colectiva, la cual se rige con fuerza normativa en tanto instituye los límites y las posibilidades de la forma en que las mujeres y los hombres actúan en el mundo (12).

Estas representaciones según Moscovici (1961; 1988;), se encuentran diferenciadas en tres clases: representaciones hegemónicas (tienden a ser uniformes y coercitivas, y prevalecen en las prácticas emocionales, afectivas y simbólicas), representaciones emancipadas (derivadas del movimiento de los saberes e ideas en subgrupos), las representaciones polémicas (enunciadas a través de la aceptación gradual, la resistencia o la discrepancia, y suelen ser establecidas a través de problemas sociales) (13).

El abordaje de las RS posibilita entender la dinámica de las interacciones sociales y aclarar los determinantes de las prácticas sociales, pues la representación, el discurso y la práctica se generan mutuamente (12).

Todo lo mencionado anteriormente, nos lleva a explorar las Representaciones Sociales acerca del autoexamen de mama de las mujeres de las zonas rurales de Risaralda, frente a la detección temprana del cáncer de mama. 


\section{MATERIALES Y MÉTODOS}

Diseño: es una investigación cualitativa de tipo exploratorio, que se va a realizar a partir de una entrevista semiestructurada aplicada a mujeres campesinas en la que a través de la comprensión de sus narrativas se puede obtener información acerca del autoexamen exploratorio como método para detectar cualquier anomalía, lo cual se contrastará teóricamente a través de las teorías de las representaciones sociales de Serge Moscovici.

Población: la muestra va a estar compuesta por 30 mujeres entre los 18 y 75 años residentes en distintas zonas rurales del departamento de Risaralda, entre los estratos 1 y $3 \sin$ antecedente diagnóstico de $\mathrm{Ca}$, de cualquier escolaridad y estado civil o creencia religiosa. La muestra va ser aleatoria de tipo intencional, y la aplicación de instrumentos debe ser consentida por parte de los entrevistados.

Técnicas de instrumentos: la técnica de recolección de la información se va a realizar a través de una entrevista semiestructurada, previa firma del consentimiento informado y la aplicación de una ficha sociodemográfica, a fin de obtener datos de caracterización. La entrevista brindará información acerca del conocimiento, percepciones e ideas asociadas al autoexamen de mama.

Validación: la validación de la información se realizará por medio de tres procedimientos: a. Indagación de diversas fuentes documentales con base en datos y la depuración de la información extraída de dichos documentos; b. la entrevista va ser revisada por personas idóneas en la temática, las cuales van a validar el instrumento; c. se va realizar una comparación de resultados obtenidos en las entrevistas con fuentes teóricas o documentales seleccionadas con anterioridad.

Procedimiento de recolección: la recolección se va a realizar con la autorización de los entrevistados a través del consentimiento informado y la aplicación de la entrevista. A los entrevistados se les van a explicar los parámetros bioéticos de la investigación, los objetivos y el alcance de la misma. La entrevista posibilita recoger de forma escrita las múltiples opiniones adscritas a las narrativas enunciados por los participantes.

Sistematización e interpretación de la información: una vez recolectada la información producto de la trascripción de las entrevistas, se procede a implementar una rejilla analítica, en la que se sistematizarán las respuestas acordes a las categorías de análisis.

El análisis se va a realizar a través de la teoría fundamentada y por procesos de codificación abierta, axial, selectiva y condicionada con base en un diseño emergente, que permite reconocer las características propias de las categorías exploradas, y también cómo en la categoría central se desenvuelven los conceptos. Finalmente se va a exportar la rejilla al software Atlas.TI a fin de realizar el análisis categorial e intertextual. 


\section{RESULTADOS}

Los resultados que se pretenden obtener de esta investigación, puesto que aún se encuentra en curso, son: la identificación de los procesos de formación de las representaciones sociales que tienen las mujeres de zonas rurales de Risaralda frente a su corporeidad (mamas), la enfermedad y el autocuidado (Autoexamen), y estar al tanto con los tipos de representaciones sociales presentes en la población estudiada, procurando la clasificación de estas para obtener perspectivas amplias de su conceptualización como individuos de comunidad. 
1. OMS (Organización Mundial de la Salud). Temas de Salud- Cáncer [Internet]. 2019. Available from: https://www.who.int/topics/cancer/es/

2. Calderón MI, Orozco MC, Mendoza GH, Alvarez C. Boletín legislativo \& político. En conmemoración al día mundial del cáncer de mama. 2018; Available from: http://www.cancer.gov.co/files/libros/archivos/boletin especial cancer de mama.pdf

3. OPS (Organización Panamericana de la salud). Cáncer de mama [Internet]. 2019. Available from: https://www.paho.org/hq/index.php?option=com_conte nt\&view $=$ article\&id $=5041: 2011$-breast-cancer $\&$ Itemid $=3639 \&$ lang $=$ es

4. Merchán A, Sánchez P, Soler LA, Valderrama F, Moreno A, Ramírez P, et al. Boletín de información Técnica Especializada de la Cuenta de Alto Costo Cáncer de mama. Cuenta Alto Costo [Internet]. 2017;3(17). Available from: www.cuentadealtocosto.org

5. CAC (Cuenta de Alto Costo). Situación del cáncer en la población adulta atendida en el SGSSS de Colombia. Bogotá; 2017. Available from: www. cuentadealtocosto.org

6. OMS (Organización Mundial de la Salud). Cáncer de mama: prevención y control [Internet]. 2019. Available from: https://www.who.int/topics/cancer/ breastcancer/es/

7. World Health Organization. Resumen De Conocimientos Prevención: Factores De Riesgo Y Prevención. Breast Heal Glob Initiat. 2016;1-12.

8. T, Hernández, FSClaudet P. Consecuencias psicoafectivas y sociales del cáncer de mama y de la mastectomía. Rev Costarric Ciencias [Internet]. 1985;6(2)(9):29-38. Available from: http://www.binasss.sa.cr/revistas/rccm/ v6n2/art4.pdf

9. Giraldo Mora CV, Arango Rojas ME. Representaciones sociales frente al autocuidado en la prevención del cáncer de mama. Investig y Educ en Enfermería [Internet]. 2009; XXVII:191-200. Available from: http://www. scielo.org.co/scielo.php?script=sci\%7B_\%7Darttext\%7B\&\%7Dpid=S0120$\underline{53072009000200004 \% 7 \mathrm{~B} \& \% 7 \mathrm{Dlang}=\mathrm{pt}}$

10. Torregrosa Jiménes R. Reflexiones Teórico-Metodológicas sobre la teoria de las representaciones sociales. Un debate necesario en Colombia. Rev Repub. 2012;(13):165-87 p.

11. Moscovici S. El Psicoanalisis, su imagen y su público. 1ed. Buenos Aires: Editorial Huemul; 1979.

12. Araya Umaña S. Las representaciones sociales: Ejes teóricos para su discusión. Facultad Latinoamericana de Ciencias Sociales (FLACSO). Costa Rica; 2002. 9-79 p.

13. Moscovici S. Psicología social, II: pensamiento y vida social; psicología social y problemas sociales. Barcelona: Editorial Paidós; 1988. 367-747 p. 